\title{
CONCEPTUAL DESIGN OF MODULAR MULTI FUNCTIONAL AGRICULTURAL MOBILE ROBOT
}

\author{
Aldis Pecka, Vitalijs Osadcuks \\ Latvia University of Life Sciences and Technologies, Latvia \\ aldis.pecka@llu.lv
}

\begin{abstract}
In order to improve competitiveness in today's business environment, farmers also have to think about the rapid technological development in their enterprises. The most important factor that can positively influence the progress of the agricultural sector is the application of modern, efficient and labor-saving technologies at the various stages of crop growing and processing. Introduction of robotics in the farming processes emerges as one of the options. Current state of technologies allows only a few tasks processed by robots to be technologically and economically viably; the most relevant example is lawn mowing robots. One of the issues that prevents widespread use of robots in agriculture is that there are still no regulations or standards in the robotic sector for the industry to allow each robot manufacturer to follow them, such as mountable equipment communication protocols, dimensions of chassis and body, control systems including sensors and actuators etc. Each robot developer chooses his own approach and interpretation in robot protocols and modules. This article presents a conceptual design of mobile robot "Formica 01" for various agricultural applications. A prototype of the proposed design has been developed and in the article various cases of its usage on strawberry fields have been described.
\end{abstract}

Key words: agricultural, mobile robot, modular.

\section{Introduction}

Food demand in the world is increasingly growing, especially it is relevant for traditional European-type food. This demand will require the use of agricultural land more effectively, mitigating environmental impacts (National Farmers’ Federation, 2013). Some of the negative factors are pollution from fossil fuels and other technical liquids used in engines and transmission, land compaction created by using heavy tractor units, problems arising from monoculture crop growing (land degradation and extensive use of pest and disease fighting chemicals) (Llewellyn \& Demden, 2010). One of the future perspectives is the introduction of mobile robots in agriculture, where groups or even swarms of smaller robots interact to process, inspect or harvest crops. Agricultural robots are forced to operate in highly stochastic environment. Changing environmental conditions, such as terrain, road surfaces, ambient lighting, biological objects, which can change color, height, volume and other parameters, pose challenges for mobile robot technology (Kim et al., 2011). To enable robot technology to enter the agricultural sector, it must be constructively simple, highly reliable, flexible and, last but not least, inexpensive. Consequently, it poses a challenge for robot developers to develop various technical solutions. Mobile robot solutions are not widely available on the market today. The most popular and most affordable ones are feeding robots in barns (Lely Juno 100), lawn mowers (Husqvarna Automower) (Grossi et al., 2016) and drones for field imaging in precision agriculture. Scientific articles discuss numerous mobile robot designs. Well- known players in the agricultural industry like John Deere, Bosh, Monsanto have also made large investments in the development of agricultural robots (Wolfert,
Verdouw, \& Bogaardt, 2017). In automation of greenhouse operations, where the environment is more predictable, stationary industrial robots in the form of CNC machines are used. Consumer mobile robots are still in their development stages (Reddy, 2016). The aim of the study is to develop conceptual design of mobile robot "Formica 01 " for various agricultural applications, such as inspection, transport, sprayer, weed destruction etc. based on selected similar designs found in literature and compare them.

\section{Materials and Methods}

Mechanical design and drive. The mechanical construction is based on a four-wheel base, divided into two drive modules each consisting of motors and gearing for two wheels. The blocks are independent and manageable separately, see image below in Figure 1. Each driving wheel has one $350 \mathrm{~W}$ brushed DC motor at nominal voltage $24 \mathrm{~V}$. Motors have built-in spur gear transmission with ratio 1:9.78, which gives $10 \mathrm{Nm}$ torque and $306 \mathrm{rpm}$ revolution speed at motor axis. Motor axis and wheels are coupled by means of chain transmission with ration 1:4 resulting in the total gear ratio of 1:39 and $40 \mathrm{Nm}$ and $76 \mathrm{rpm}$ on the axis of wheel. Robot wheels are with camera tires and with diameter of $400 \mathrm{~mm}$, with maximum load $200 \mathrm{~kg}$ on each; maximum speed is $10 \mathrm{~km} \cdot \mathrm{h}^{-1}$. Self-weight of the robot is $100 \mathrm{~kg}$, full weight is $300 \mathrm{~kg}$. With full payload robot can overpass road rise of up to 35 degrees. Each wheel is operated at constant speed. To enable the robot to maneuver, different speed is set to wheels on each side. The principle is the same as for caterpillar tractor.

Space between the drive modules is dedicated to the frame with power source (battery unit), control system module and application-specific equipment 

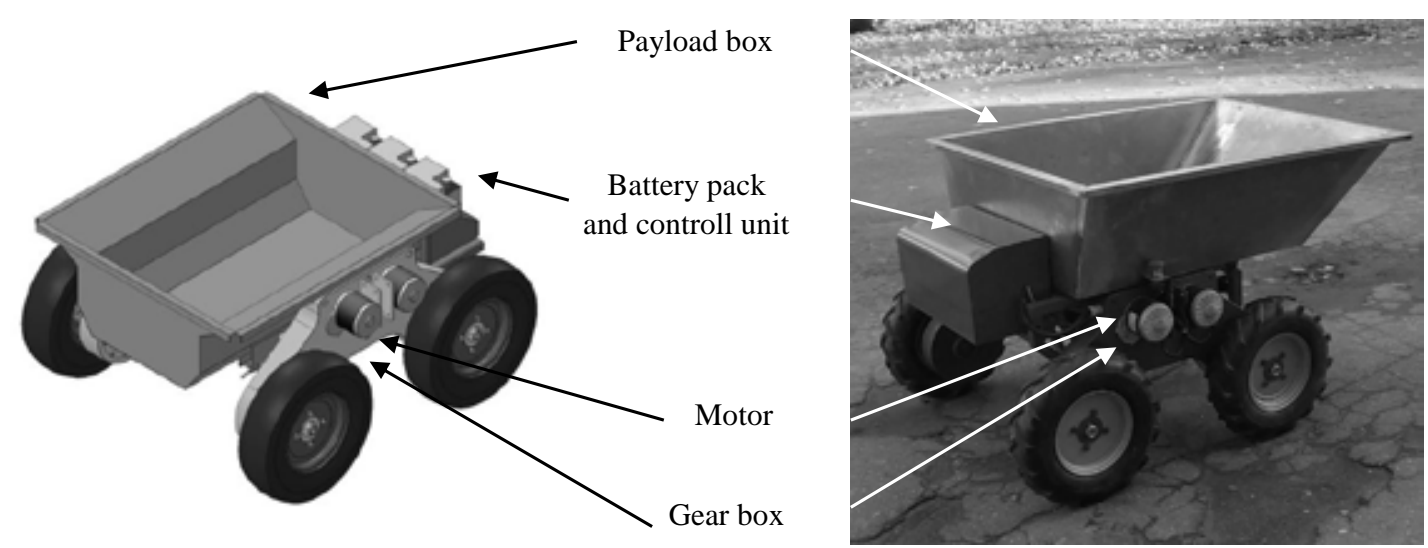

Figure 1. Mechanical construction of "Formica 01 ".

(trolley, soil sampler, weed fighting unit etc.). The drive modules are designed so that they can be easily detached from the central frame using screws; and application equipment then can be changed. From this also comes the strength of the proposed design. It is possible to adjust easily the track width to make possible the operation of robot along plant rows with different sizes.

Power source and electrical system design. Lead-acid batteries are used as a power source. Two batteries are connected in series with the total voltage of $24 \mathrm{~V}$ and average energy charge 960 Wh. Using this battery setup the robot can go on asphalt road at the maximum speed of approximately $0.5 \mathrm{~h}$. One of the reasons using lead-acid batteries instead of lithium-based ones is the possibility to charge and discharge them at temperatures below $-10^{\circ} \mathrm{C}$, without significant loss in capacity. Besides battery unit the frame between drive modules holds the main control module, which consists of motor driver power stages, electronic control unit (ECU) for the motor, lighting, external equipment control and sensor reading, motor drivers and single board computer Raspberry PI for high level control (localization, path planning,
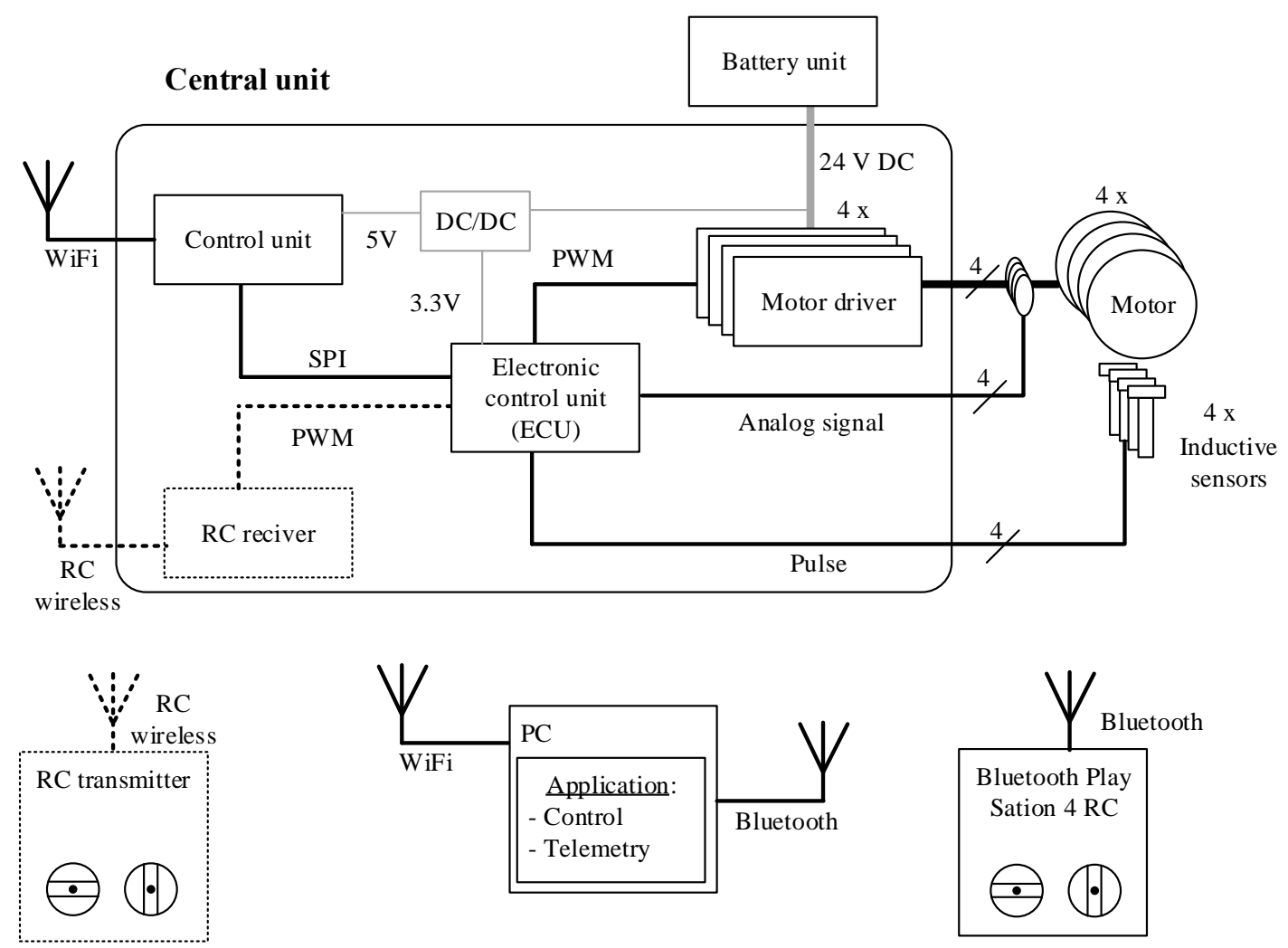

Figure 2. Mechanical construction of the mobile robot. 


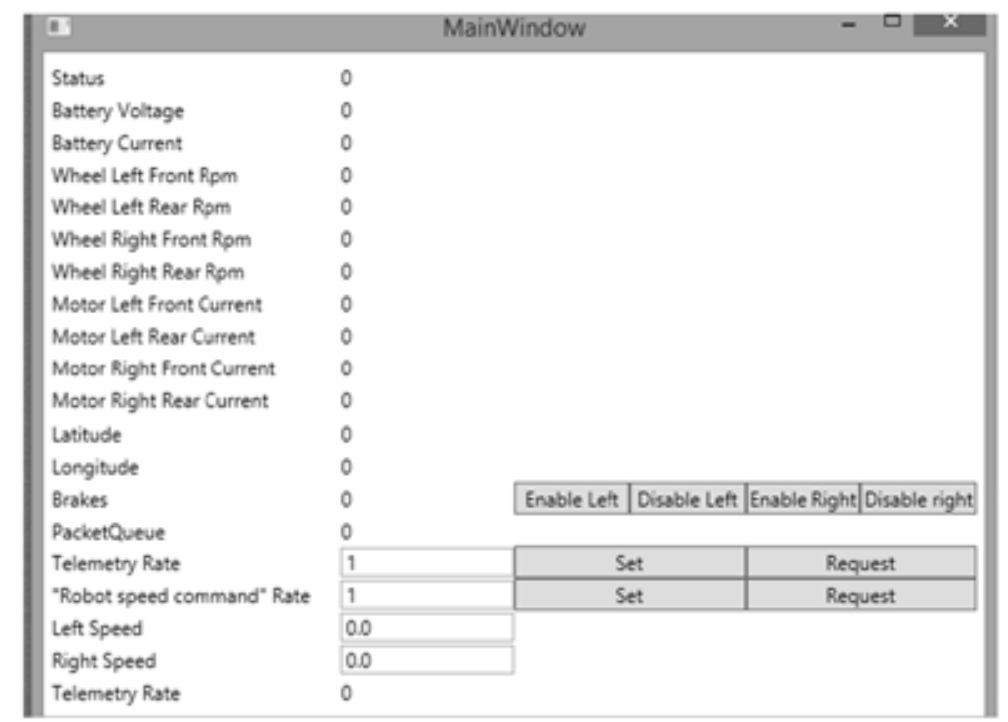

Figure 3. Mobile robot control and monitoring interface on PC.

obstacle avoidance, human or GPS path following etc.), see Figure 2.

Motor speed and direction is controlled by Cytron 30A motor driver board, consisting of discrete power MOSFETs, gate drivers and logic circuits for direction change. Rated voltage is $24 \mathrm{~V}$ and current - $30 \mathrm{~A}$ (up to $80 \mathrm{~A}$ for $1 \mathrm{~s}$ pulse). One driver board per motor is used. Cytron 30A has two inputs: PWM signal for speed control and logical signal for rotating direction change. The driver boards are controlled directly from ECU. Feedback signal from each wheel speed is obtained from NPN-type industrial inductive sensors and tooth disk. To get better speed, the resolution disk is placed on the motor axis and has 60 teeth. An inductive sensor was used instead of possible alternatives, like optocouplers, to increase the reliability of reading. Constant rotation speed is controlled using PID controller. The current of each motor is measured using ACS712 Hall effect sensor. Its range is $\pm 30 \mathrm{~A}$ which is sufficient for the motors used.

ECU has two interfacing options for a higher-level control system, only one can be used at a time. One is intended for accepting signals from the standard remote control (RC) receiver. Thus the conventional remote control unit for RC models or drones can be used. Each stick of the RC unit is used for speed and direction control of the respective (left and right) drive module. The other interface is SPI which accepts commands and returns telemetry data form sensors. Telemetry data includes rotational speed of each wheel, current of each motor and current and voltage of batteries.

Raspberry PI microcomputer is used a higherlevel controller. The computer runs on a Linux-based operating system and has multiple interfaces to external devices: SPI (used for communication with robot's main ECU), Etherntet, WiFi, USB and general purpose digital IO. The computer has Quad Core 1.2GHz Broadcom BCM2837 64bit CPU, 1 GB RAM and ROM on detachable microSD card for operating system.

The computer communicates with external devices using WiFi interface as an access point. It is done by TCP socket server software running on Raspberry PI, which accepts commands from a client on a remote computer in real time, decodes and transfers them to ECU. This way literally all types of user interfaces can be used, for example, standard Bluetooth game controller. Needless to say that commands can be sent also from software on a personal computer now acting as a remote controller. Screenshot of user application of telemetry software is shown in Figure 3.

\section{Results and Discussion}

A prototype of the proposed mobile agricultural robot design has been developed. Emphasis in this design was laid on its easy adaptability for performing various tasks. One of the tasks, on which it has been successfully approved, was transporting strawberry crates on fields. This way human workers do not need to bring crates themselves and can concentrate on a more complicated task of harvesting the berries, see Figure 4.

Another example is transporting of various cargo using trolley seen in Figure 1. Another task planned, but not experimentally validated yet, is grass mowing application for farming needs (e.g. trail cleaning between strawberry rows or in fruit gardens). For this task between drive modules a mowing plate with blades and dedicated motor should be used instead of a base frame.

Raspberry PI computer used as the main control unit for the mobile agricultural robot is a cost effective way of bringing together or fusing data from various 


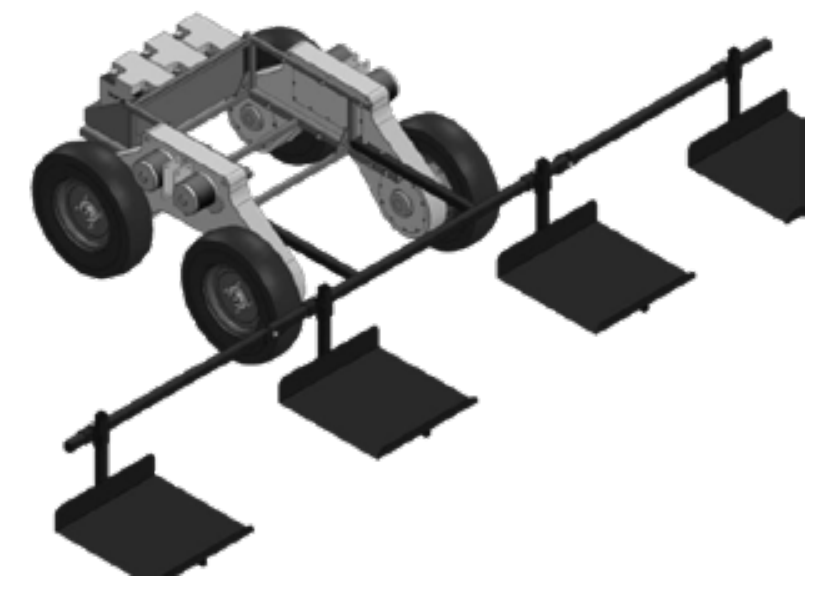

Figure 4. Mobile robot on strawberry field.

Table 1

Shows other design concepts in comparison with the created mobile agricultural robot

\begin{tabular}{|c|c|c|c|c|c|}
\hline Nr. & Parameter & Formica 01 & $\begin{array}{l}\text { Agricultural Mobile } \\
\text { Robot Fig 4.a } \\
\text { (Tabile et al., 2011) }\end{array}$ & $\begin{array}{l}\text { AgBot II Fig 4.b } \\
\text { (Bawden et al., } \\
\text { 2011) }\end{array}$ & $\begin{array}{l}\text { BoniRob Fig 4.c } \\
\text { (Fleckenstein, } \\
\text { Dornhege, \& } \\
\text { Burgard, 2017) }\end{array}$ \\
\hline 1. & $\begin{array}{l}\text { Agriculture } \\
\text { tasks }\end{array}$ & Multiple tasks & Multiple tasks & $\begin{array}{l}\text { Inspection, } \\
\text { sprayer, weed } \\
\text { destruction }\end{array}$ & Multiple tasks \\
\hline 2. & $\begin{array}{l}\text { Steering, } \\
\text { driving }\end{array}$ & $\begin{array}{l}\text { Differential steering system } \\
\text { with each motor on wheel }\end{array}$ & $\begin{array}{l}\text { Articulated steering } \\
\text { with each motor on } \\
\text { wheel }\end{array}$ & $\begin{array}{l}\text { Front arms can be } \\
\text { moved separately } \\
\text { rear wheels for } \\
\text { driving }\end{array}$ & $\begin{array}{l}\text { Each of the arms can } \\
\text { be moved separately } \\
\text { with each motor on } \\
\text { wheel }\end{array}$ \\
\hline 3. & $\begin{array}{l}\text { Operational } \\
\text { speed }\end{array}$ & $10 \mathrm{~km} \mathrm{~h}^{-1}$ & - & $5 \mathrm{~km} \mathrm{~h}^{-1}$ & $8 \mathrm{~km} \mathrm{~h}^{-1}$ \\
\hline 4. & Payload mass & $200 \mathrm{~kg}$ & - & $200 \mathrm{~kg}$ & $150 \mathrm{~kg}$ \\
\hline 5. & Vehicle mass & $100 \mathrm{~kg}$ & - & $500 \mathrm{~kg}$ & $400 \mathrm{~kg}$ \\
\hline 6. & Wheel width & $0.4 \mathrm{~m}$ & $0.4 \mathrm{~m}$ & $0.3 \mathrm{~m}$ & $0.4 \mathrm{~m}$ \\
\hline 7. & Track gauge & $\begin{array}{l}\text { Manually changed, depends } \\
\text { on central frame dimensions }\end{array}$ & Unchanged & Unchanged & $\begin{array}{l}\text { Adaptable, from } 750 \\
\text { to } 2000 \mathrm{~mm}\end{array}$ \\
\hline
\end{tabular}

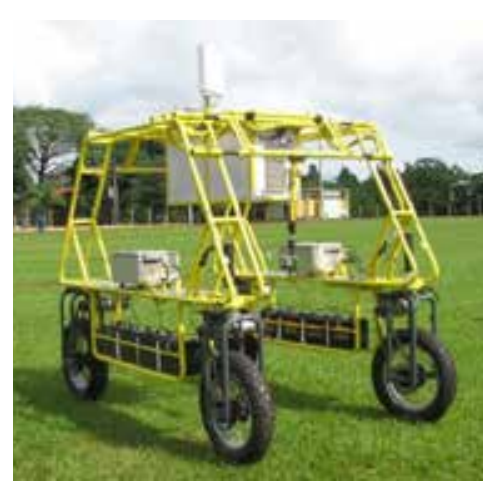

a) Agricultural Mobile Robot

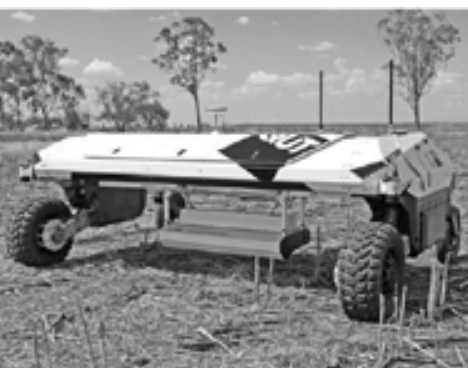

b) AgBot II

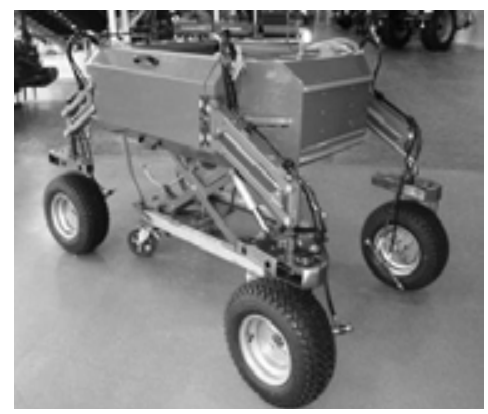

c) BoniRob

Figure 5. Agricultural mobile robots. 
environmental sensors. An extensive library of a third party software for interfacing with optical, IR and event-based cameras, GNSS receivers, LIDARs, LTE modems and more is readily available. As Linux OS is used, there is a wide range of software development options for native and managed or, scripting environments: GCC compiler, Java, Mono environment, Python etc.

\section{Conclusions}

1. Modular design of mobile agricultural robot has been developed aiming to increase the number of tasks that could be accomplished by a single robotized unit, thus increasing its economic effectiveness with a changeable central frame to use in multiple tasks.

2. The robot is tested in a real farm in ZS "Rubeni" and the user's conclusion are:

a. A prototype unit of the proposed design has been developed and its ability to perform simple transportation tasks has been approved in real situations: on a raspberry field and general transportation tasks on small to medium farms. b. The mobile robot platform can also be used in other areas, such as paving, construction, removal of trees from the forest, etc.

3. The popular Raspberry Pi platform as a central control unit allows the use of various interfacing options to do the sensing, localization image processing and using other equipment, as well as readily available algorithms for general mobile robot control.

4. The proposed agricultural robot design addresses such drawbacks found in other solutions as: too heavy weight and, consequently, pressure on ground, slow movement speed and mechanical construction, which is hardly adjustable for multiple applications with a different width of plant rows.

\section{Acknowledgements}

The authors would like to express their gratitude for the financial support from project "Zinātniskās kapacitātes stiprināšanas LLU” nr. Z18.

\section{References}

1. Bawden, O., Kulk, J., Russell, R., McCool, C., English, A., Dayoub, F., Lehnert, C., \& Perez, T. (2017). Robot for weed species plant-specific management. Wiley Online Library, DOI: 10.1002/rob.21727.

2. Fleckenstein, F., Dornhege, C., \& Burgard, W. (2017). Efficient path planning for mobile robots with adjustable wheel positions. Proceedings - IEEE International Conference on Robotics and Automation, 21 July 2017 (pp. 2454-2460). Germany: University of Freiburg.

3. Grossi, N., Fontanelli, M., Garramone, E., Peruzzi, A., Raffaelli, M., Pirchio, M., Martelloni, L., Frasconi, C., Caturegli, L., Gaetani, M., Magni, S., Scott McElroy, J., \& Volterrani, M. (2016). Autonomous mower saves energy and improves quality of tall fescue lawn [Abstract]. HortTechnology. 26(6), 825-830. DOI: 10.21273/HORTTECH03483-16.

4. Kim, K.-O., Kim, J.-C., Ban, K.-J., Kim, E.-K., \& Jang, M.-S. (2011). U-IT based greenhouse environment monitoring system. Proceedings of the 2011 5th FTRA International Conference on Multimedia and Ubiquitous Engineering, 4 Mar 2011 (pp. 203-206). Loutraki, Greece: IEEE Explore digital Library.

5. Llewellyn, R.S., \& Demden, F.H. (2010). Adoption of no-till cropping practices in australian grain growing regions. Australia: Australia Government Publishing Service.

6. National Farmers Federation. (2013). The blueprint for australian agriculture 2013-2020. Australia: Australia Government Publishing Service.

7. Reddy, N.V., Reddy, A.V.V.V., Pranavadithya, S., \& Kumar, J.J. (2016). A critical review on agricultural robots [Abstract]. International Journal of Mechanical Engineering and Technology, 7 (4), 183-188.

8. Tabile, R.A., Godoy, E.P., Pereira, R.R.D., Tangerino, G.T., Porto, A.J.V., \& Inamasu, R.Y. (2011). Design and development of the architecture of an agricultural mobile robot. Journal Engenharia Agricola, 31 (1), $130-142$.

9. Wolfert, S., Ge, L., Verdouw, C., \& Bogaardt, M.-J. (2017). Big Data in Smart Farming - A review [Abstract]. Agricultural Systems, 153, 69-80. DOI: 10.1016/j.agsy.2017.01.023. 